\title{
Quadratic functionals on modules over complex Banach $*$-algebras with an approximate identity
}

\author{
by \\ DiJAna ILIŠEvić (Zagreb)
}

\begin{abstract}
The problem of representability of quadratic functionals by sesquilinear forms is studied in this article in the setting of a module over an algebra that belongs to a certain class of complex Banach $*$-algebras with an approximate identity. That class includes $\mathrm{C}^{*}$-algebras as well as $\mathrm{H}^{*}$-algebras and their trace classes. Each quadratic functional acting on such a module can be represented by a unique sesquilinear form. That form generally takes values in a larger algebra than the given quadratic functional does. In some special cases, such as when the module is also a complex vector space compatible with the vector space of the underlying algebra, and when the quadratic functional is positive definite with values in a $\mathrm{C}^{*}$-algebra or in the trace class for an $\mathrm{H}^{*}$-algebra, the resulting sesquilinear form takes values in the same algebra. In particular, every normed module over a $\mathrm{C}^{*}$-algebra, or an $\mathrm{H}^{*}$-algebra, without nonzero commutative closed two-sided ideals is a pre-Hilbert module. Furthermore, the representation theorem for quadratic functionals acting on modules over standard operator algebras is also obtained.
\end{abstract}

Introduction. Let $M$ be a right module over a $*$-ring $R$. Then a mapping $Q: M \rightarrow R$ is called a quadratic functional if it satisfies the parallelogram law

$$
Q(f+g)+Q(f-g)=2 Q(f)+2 Q(g) \quad \text { for all } f, g \in M
$$

and the homogeneity equation

$$
Q(f a)=a^{*} Q(f) a \quad \text { for all } f \in M \text { and } a \in R .
$$

A biadditive form $S: M \times M \rightarrow R$ is called sesquilinear if

$$
S(f a, g)=a^{*} S(f, g), \quad S(f, g a)=S(f, g) a \quad \text { for all } f, g \in M \text { and } a \in R .
$$

For every sesquilinear form $S: M \times M \rightarrow R$, the mapping $Q: M \rightarrow R$ defined by $Q(f)=S(f, f)$ is a quadratic functional. The question is if each

2000 Mathematics Subject Classification: Primary 46L, 46K15, 46K50, 47A07, 46H25, 46L08; Secondary 16W, 39B52, 47B47.

Key words and phrases: Banach *-algebra, approximate identity, $\mathrm{C}^{*}$-algebra, $\mathrm{H}^{*}$ algebra, trace class, standard operator algebra, right module, pre-Hilbert module, normed module, quadratic functional, sesquilinear form, Jordan $*$-derivation, double centralizer. 
quadratic functional can be represented by some sesquilinear form. More precisely, for a given quadratic functional $Q: M \rightarrow R$, does there exist a sesquilinear form $S: M \times M \rightarrow R$ satisfying $S(f, f)=Q(f)$ for every $f \in M$ ?

The solution of this problem is closely connected with the structure of certain mappings, called Jordan $*$-derivations, on the underlying ring. An additive mapping $J$ defined on $R$ is called a Jordan $*$-derivation if it satisfies

$$
J\left(a^{2}\right)=a^{*} J(a)+J(a) a \quad \text { for every } a \in R .
$$

For every $u \in R$, the mapping $J_{u}: R \rightarrow R$ given by $J_{u}(a)=u a-a^{*} u$ is an example of a Jordan $*$-derivation and it is called an inner Jordan *derivation. (Our definition of a Jordan $*$-derivation is, in a manner, reversed in comparison with the one in [12] because we deal with right modules instead of left ones. Therefore, the statements of some cited results are adjusted to our case.)

Representability of quadratic functionals acting on modules over $*$-rings with identity is dealt with in the Main Theorem of [13]. A simple consequence of that result is the positive solution of the representability problem for modules over any complex $*$-algebra with identity. It is based on the easily verified fact that each Jordan $*$-derivation on a complex $*$-algebra with identity is inner and uniquely represented. If we omit the assumption of the existence of the identity, the class of algebras has to be restricted (observe that the mapping $J: A \rightarrow A$ defined by $J(a)=a-a^{*}$ is a noninner Jordan *-derivation if $A$ is a nonunital complex $*$-algebra). We are motivated by the fact that closest to the algebras with identity are the algebras with an approximate identity, and by the technique dealing with an approximate identity consisting of projections, developed in [17]. In this paper we study quadratic functionals on modules over a certain class of complex Banach *-algebras with an approximate identity.

We use the term Banach *-algebra for a *-algebra $A$ together with a complete submultiplicative norm $\|\cdot\|$ such that $\left\|a^{*}\right\|=\|a\|$ for every $a \in A$. An approximate identity for $A$ is a generalized sequence $\left\{e_{\alpha}\right\}$ of elements of $A$ having the property

$$
\lim _{\alpha}\left\|e_{\alpha} a-a\right\|=\lim _{\alpha}\left\|a e_{\alpha}-a\right\|=0 \quad \text { for every } a \in A .
$$

Obviously, if $\left\{e_{\alpha}\right\}$ is an approximate identity for $A$, then so is $\left\{e_{\alpha}^{*}\right\}$.

In Section 1 it is shown how Jordan $*$-derivations of a large class of complex $*$-algebras can be understood as inner ones in the context of double centralizers. Section 2 contains the main results concerning representability of quadratic functionals acting on modules over complex Banach $*$-algebras with an approximate identity. They show the closeness to the corresponding result in the case of complex $*$-algebras having an identity element. In 
Section 3 the representation theorem is established for quadratic functionals on modules over standard operator algebras. Section 4 is devoted to an application of the results of the previous sections to normed modules over $\mathrm{H}^{*}$-algebras and $\mathrm{C}^{*}$-algebras.

1. Jordan $*$-derivations and double centralizers. Let $A$ be a ring (resp. an algebra). If the mappings $T, S: A \rightarrow A$ satisfy $a T(b)=S(a) b$ for all $a, b \in A$, then the pair $(T, S)$ is called a double centralizer of $A$. For $a \in A$ we denote by $L_{a}$ the left multiplication operator (defined by $L_{a}(x)=a x$ for every $x \in A$ ) and by $R_{a}$ the right multiplication operator (which is given by $R_{a}(x)=x a$ for every $x \in A$ ); then $\left(L_{a}, R_{a}\right)$ is an example of a double centralizer of $A$.

Let us define:

$$
\begin{aligned}
& \mathcal{L}(A)=\left\{L_{a}: a \in A\right\}, \\
& L_{\mathrm{DC}}(A)=\{T: A \rightarrow A: \text { there exists } S: A \rightarrow A \text { such that } \\
&(T, S) \text { is a double centralizer of } A\}, \\
& L(A)=\{T: A \rightarrow A: T(a b)=T(a) b \text { for all } a, b \in A\} .
\end{aligned}
$$

It is easy to verify that $\mathcal{L}(A), L_{\mathrm{DC}}(A)$ and $L(A)$ are rings (resp. algebras). Let us observe that $L_{\mathrm{DC}}(A)$ is the ring (resp. the algebra) of all "the left halves of double centralizers". Since $\left(L_{a}, R_{a}\right)$ is a double centralizer of $A$ for every $a \in A$, we have $\mathcal{L}(A) \subseteq L_{\mathrm{DC}}(A)$. Note that $L_{\mathrm{DC}}(A)$ has an identity. If $A$ does not admit a left identity, then $\mathcal{L}(A) \subsetneq L_{\mathrm{DC}}(A)$.

REMARK 1.1. If $A$ is a ring such that $A a=0$ (where $a \in A$ ) implies $a=0$, then $L_{\mathrm{DC}}(A) \subseteq L(A)$. This is in fact the statement of Observation 13 in [17], placed in the context of $\mathrm{H}^{*}$-algebras. However, it holds in this more general setting as can be seen from the proof in [17]. Furthermore, if $A$ is an $\mathrm{H}^{*}$-algebra, then $L_{\mathrm{DC}}(A)=L(A)$, but there is a $\mathrm{C}^{*}$-algebra $A$ such that $L_{\mathrm{DC}}(A) \subsetneq L(A)$ (for more details see the Remark and Example in [6]).

REMARK 1.2. Following the proof of Observation 13 in [17], we can also deduce that if $A$ is a Banach algebra having the property that $A a=0$ (where $a \in A$ ) implies $a=0$, then each element in $L_{\mathrm{DC}}(A)$ is a bounded linear operator.

The following result shows that each Jordan $*$-derivation acting on a certain complex $*$-algebra is "close enough" to an inner one and can be handled analogously.

Lemma 1.3. Let $A$ be a complex $*$-algebra such that $A a=0$ (where $a \in A$ ) implies $a=0$ (which is equivalent to $a A=0$ implying $a=0$ ). For each Jordan $*$-derivation $J: A \rightarrow A$ there exists a unique $T \in L_{\mathrm{DC}}(A)$ such that $L_{J(a)}=T L_{a}-L_{a^{*}} T$ for every $a \in A$. 
Proof. For each Jordan $*$-derivation $J: A \rightarrow A$ there exists a unique double centralizer $(T, S)$ of $A$ such that $J(a)=T(a)-S\left(a^{*}\right)$ for every $a \in A$ ([2, Theorem 2.1]). For all $a, b \in A$,

$$
\begin{aligned}
L_{J(a)}(b) & =J(a) b=\left(T(a)-S\left(a^{*}\right)\right) b=T(a) b-S\left(a^{*}\right) b \\
& =T(a) b-a^{*} T(b) \stackrel{\text { Rem. } 1.1}{=} T(a b)-a^{*} T(b) \\
& =T L_{a}(b)-L_{a^{*}} T(b)=\left(T L_{a}-L_{a^{*}} T\right)(b) .
\end{aligned}
$$

Therefore, $L_{J(a)}=T L_{a}-L_{a^{*}} T$ for every $a \in A$.

If $T_{1} \in L_{\mathrm{DC}}(A)$ satisfies $L_{J(a)}=T_{1} L_{a}-L_{a^{*}} T_{1}$ for every $a \in A$ and if $S_{1}: A \rightarrow A$ is the mapping such that $x T_{1}(y)=S_{1}(x) y$ for all $x, y \in A$, then

$$
\begin{aligned}
& J(a) b=L_{J(a)} b=\left(T_{1} L_{a}-L_{a^{*}} T_{1}\right)(b)=T_{1}(a b)-a^{*} T_{1}(b) \\
& \stackrel{\text { Rem.1.1 }}{=} T_{1}(a) b-a^{*} T_{1}(b)=T_{1}(a) b-S_{1}\left(a^{*}\right) b=\left(T_{1}(a)-S_{1}\left(a^{*}\right)\right) b
\end{aligned}
$$

for all $a, b \in A$. Hence, $J(a)=T_{1}(a)-S_{1}\left(a^{*}\right)$ for every $a \in A$. The uniqueness of such a representation implies $T_{1}=T$.

Remark 1.4. Clearly, if $J: A \rightarrow A$ is an inner Jordan *-derivation, that is, if there exists $u \in A$ such that $J(a)=u a-a^{*} u$ for every $a \in A$, then the unique $T \in L_{\mathrm{DC}}(A)$ obtained in Lemma 1.3 is equal to $L_{u}$.

2. The representation theorem and its consequences. The main theorem of this article is Theorem 2.1. Although it is related to a class of Banach $*$-algebras, it is algebraic in spirit.

Theorem 2.1. Let $(A,\|\cdot\|)$ be a complex Banach *-algebra with an approximate identity $\left\{e_{\alpha}\right\}$ such that

$$
\lim _{\alpha}\left\|e_{\alpha}{ }^{*} a e_{\alpha}-a\right\|=0 \quad \text { for every } a \in A .
$$

Let $M$ be a right module over $A$. For each quadratic functional $Q: M \rightarrow A$ there exists a unique mapping $S: M \times M \rightarrow L_{\mathrm{DC}}(A)$ satisfying:

(i) $S$ is biadditive,

(ii) $S(f a, g)=L_{a^{*}} S(f, g)$ and $S(f, g a)=S(f, g) L_{a}$,

(iii) $S(f, f)=L_{Q(f)}$,

for all $f, g \in M$ and $a \in A$. This mapping is given by

$$
\begin{aligned}
S(f, g) a= & \frac{1}{4}(Q(f+g) a-Q(f-g) a) \\
& +\frac{i}{4} \lim _{\alpha}\left(Q\left(f\left(i e_{\alpha}\right)+g\right) a-Q\left(f\left(i e_{\alpha}\right)-g\right) a\right)
\end{aligned}
$$

for all $f, g \in M$ and $a \in A$.

Remark 2.2. Observe that $A a=0$ (where $a \in A$ ) implies $a=0$, as well as $a A=0(a \in A)$ implies $a=0$, since $A$ has an approximate identity. 
Remark 2.3. If we identify $A$ with $\mathcal{L}(A)$, then $M$ can be understood as a module over $\mathcal{L}(A)$. Therefore, we can say that $S$ is a sesquilinear form on $M \times M$, not taking values in $\mathcal{L}(A)$, but in the larger algebra $L_{\mathrm{DC}}(A)$.

REMARK 2.4. Let $(A,\|\cdot\|)$ be a complex Banach $*$-algebra with an approximate identity $\left\{e_{\alpha}\right\}$. If the generalized sequence $\left\{L_{e_{\alpha}^{*}}\right\}$ consisting of left multiplication operators is bounded, then

$$
\begin{aligned}
\left\|e_{\alpha}^{*} a e_{\alpha}-a\right\| & \leq\left\|e_{\alpha}^{*} a e_{\alpha}-e_{\alpha}^{*} a\right\|+\left\|e_{\alpha}^{*} a-a\right\| \\
& =\left\|L_{e_{\alpha}^{*}}\left(a e_{\alpha}-a\right)\right\|+\left\|a^{*} e_{\alpha}-a^{*}\right\| \\
& \leq\left\|L_{e_{\alpha}^{*}}\right\| \cdot\left\|a e_{\alpha}-a\right\|+\left\|a^{*} e_{\alpha}-a^{*}\right\| \rightarrow 0,
\end{aligned}
$$

so the assumptions of Theorem 2.1 are satisfied.

Examples of such algebras and approximate identities follow:

I. Clearly, $\left\{L_{e_{\alpha}^{*}}\right\}$ is bounded if $\left\{e_{\alpha}^{*}\right\}$ is bounded. This is the case when $A$ is a $\mathrm{C}^{*}$-algebra and $\left\{e_{\alpha}\right\}$ is the canonical approximate identity for $A$ (e.g. [7, Theorem 3.1.1]).

II. Every $\mathrm{H}^{*}$-algebra has an approximate identity $\left\{e_{\alpha}\right\}$ consisting of projections ([5, Theorem 3.2], based on [1, Theorem 4.1]). Corollary 3.11 of [15] implies boundedness of $\left\{L_{e_{\alpha}}\right\}$ (more precisely, $\left\|L_{e_{\alpha}}\right\|=1$ for every $\alpha$ ) although $\left\{e_{\alpha}\right\}$ generally is not bounded. (For simplicity we use the term "H*-algebra" for "proper $\mathrm{H}^{*}$-algebra".)

III. The trace class associated with an $\mathrm{H}^{*}$-algebra $A$ is defined as the set $\tau(A)=\{x y: x, y \in A\}$. It is a self-adjoint two-sided ideal of $A$ which is dense in $A$ and it can be equipped with a norm $\tau(\cdot)$ such that $(\tau(A), \tau(\cdot))$ is a Banach $*$-algebra. The inequality $\tau(x y) \leq\|x\| \cdot\|y\|$ holds for all $x, y \in A$. These facts and more details concerning the trace class can be found in [11] and [10]. An approximate identity $\left\{e_{\alpha}\right\}$, consisting of projections, for an $\mathrm{H}^{*}$-algebra is also an approximate identity for its trace class. Namely, if $a \in \tau(A)$, then there exist $x, y \in A$ such that $a=x y$, so

$$
\begin{aligned}
& \tau\left(e_{\alpha} a-a\right)=\tau\left(e_{\alpha} x y-x y\right)=\tau\left(\left(e_{\alpha} x-x\right) y\right) \leq\left\|e_{\alpha} x-x\right\| \cdot\|y\| \rightarrow 0, \\
& \tau\left(a e_{\alpha}-a\right)=\tau\left(x y e_{\alpha}-x y\right)=\tau\left(x\left(y e_{\alpha}-y\right)\right) \leq\|x\| \cdot\left\|y e_{\alpha}-y\right\| \rightarrow 0 .
\end{aligned}
$$

Note that $e_{\alpha}=e_{\alpha}^{2} \in \tau(A)$ for every $\alpha$. Furthermore, Lemma 5 of [11] implies $\tau\left(L_{e_{\alpha}}(a)\right) \leq\left\|L_{e_{\alpha}}\right\| \cdot \tau(a)=\tau(a)$ for every $a \in \tau(A)$ and every $\alpha$; hence $\left\{L_{e_{\alpha}}\right\}$ is bounded.

Let us emphasize that a right module $M$ over an algebra $A$ is understood as a right module $M$ over the ring $A$. More precisely, it is not required that $M$ is a complex vector space. Clearly, a module over each unital complex algebra is naturally equipped with the structure of a complex vector space compatible with the structure of $A$. (Compatibility of these structures means 
that $(\lambda f) a=f(\lambda a)=\lambda(f a)$ for all $\lambda \in \mathbb{C}, f \in M$ and $a \in A$.) In contrast to that case, the following example shows that there exists a module over an arbitrary complex normed algebra with an approximate identity, but without an identity, which cannot be equipped with the structure of a complex vector space compatible with the structure of the underlying algebra. This example is inspired by the process of adjunction of an identity to an algebra without an identity.

EXAMPLE 2.5. Let $A$ be a complex normed algebra without an identity, but with an approximate identity $\left\{e_{\alpha}\right\}$. The set $M=A \times \mathbb{R}$ endowed with the coordinatewise defined addition

$$
(a, \lambda)+(b, \mu)=(a+b, \lambda+\mu) \quad \text { for all } a, b \in A \text { and } \lambda, \mu \in \mathbb{R}
$$

is a right module over $A$ under its action on $A$ defined by

$$
(a, \lambda) b=(a b+\lambda b, 0) \quad \text { for all } a, b \in A \text { and } \lambda \in \mathbb{R} .
$$

Assume that $M$ is equipped with the structure of a complex vector space compatible with the structure of $A$. Then we have

$$
(i \cdot(0,1)) e_{\alpha}=(0,1)\left(i e_{\alpha}\right), \quad \text { that is, }(i \cdot(0,1)) e_{\alpha}=\left(i e_{\alpha}, 0\right) .
$$

If we put $i \cdot(0,1)=(u, \eta) \in M$, then

$$
(i \cdot(0,1)) e_{\alpha}=(u, \eta) e_{\alpha}=\left(u e_{\alpha}+\eta e_{\alpha}, 0\right)
$$

and consequently

$$
u e_{\alpha}+\eta e_{\alpha}=i e_{\alpha}, \quad \text { that is, } u e_{\alpha}=(i-\eta) e_{\alpha} .
$$

The generalized sequence $\left\{u e_{\alpha}\right\}$ is convergent to $u \in A$, hence so also is $\left\{(i-\eta) e_{\alpha}\right\}$. For every $a \in A$,

$$
\begin{aligned}
& u a=\left(\lim _{\alpha}(i-\eta) e_{\alpha}\right) a=(i-\eta) \lim _{\alpha} e_{\alpha} a=(i-\eta) a, \\
& a u=a\left(\lim _{\alpha}(i-\eta) e_{\alpha}\right)=(i-\eta) \lim _{\alpha} a e_{\alpha}=(i-\eta) a .
\end{aligned}
$$

Evidently, $\eta \neq i$ because $\eta \in \mathbb{R}$. Hence, $\frac{1}{i-\eta} u$ is an identity in $A$, contradicting the assumption that $A$ is nonunital.

Proof of Theorem 2.1. Lemma 4 in [4] is related to the problem of representability of positive definite quadratic functionals acting on a module over an $\mathrm{H}^{*}$-algebra. However, statements (i)-(iv) and (vi)-(vii) of that lemma can be carried over to arbitrary (not necessarily positive definite) quadratic functionals on a module over any algebra satisfying the assumptions of the main theorem. Thus the mapping $B: M \times M \rightarrow A$ given by

$$
B(f, g)=Q(f+g)-Q(f-g)
$$


has the following properties:

(B1) $B(f, f)=4 Q(f)$,

(B2) $B(f, g)=B(g, f)$,

(B3) $B(f, g+h)=B(f, g)+B(f, h)$,

(B4) $B(f a, g a)=a^{*} B(f, g) a$,

(B5) $\quad B(f+f a, g+g a)=B(f, g)+a^{*} B(f, g)+B(f, g) a+B(f a, g a)$,

(B6) $B(f a, g)+B(f, g a)=a^{*} B(f, g)+B(f, g) a$,

for all $f, g, h \in M$ and $a \in A$. Let us mention that $\left\{e_{\alpha}\right\}$ appears only in the proof of (B5) where the assumption $\lim _{\alpha}\left\|e_{\alpha}^{*} a e_{\alpha}-a\right\|=0$ is used.

For fixed $f, g \in M$, the mapping $J: A \rightarrow A$ defined by

$$
J(a)=B(f a, g)-a^{*} B(f, g)
$$

satisfies

$$
\begin{aligned}
a^{*} J(a)+J(a) a & =a^{*}\left(B(f a, g)-a^{*} B(f, g)\right)+\left(B(f a, g)-a^{*} B(f, g)\right) a \\
& =\left(a^{*} B(f a, g)+B(f a, g) a\right)-\left(a^{* 2} B(f, g)+a^{*} B(f, g) a\right) \\
& \stackrel{(\mathrm{B} 6)}{=}\left(B\left(f a^{2}, g\right)+B(f a, g a)\right)-\left(a^{* 2} B(f, g)+a^{*} B(f, g) a\right) \\
& \stackrel{(\mathrm{B} 4)}{=}\left(B\left(f a^{2}, g\right)+a^{*} B(f, g) a\right)-\left(a^{* 2} B(f, g)+a^{*} B(f, g) a\right) \\
& =B\left(f a^{2}, g\right)-a^{* 2} B(f, g)=J\left(a^{2}\right) .
\end{aligned}
$$

Hence, $J$ is a Jordan $*$-derivation. According to Lemma 1.3 , there exists a unique $T^{f, g} \in L_{\mathrm{DC}}(A)$ such that

$$
L_{B(f a, g)}-L_{a^{*} B(f, g)}=T^{f, g} L_{a}-L_{a^{*}} T^{f, g} \quad \text { for every } a \in A .
$$

Let $C: M \times M \rightarrow L_{\mathrm{DC}}(A)$ be the mapping given by

$$
C(f, g)=T^{f, g} .
$$

Then

(BC) $\quad L_{B(f a, g)}-L_{a^{*} B(f, g)}=C(f, g) L_{a}-L_{a^{*}} C(f, g)$

for all $f, g \in M$ and $a \in A$. Let us prove that the mapping $C$ has the following properties:

(C1) $C(f, g)+C(g, f)=L_{B(f, g)}$, specifically $C(f, f)=2 L_{Q(f)}$,

(C2) $\quad C(f+g, h)=C(f, h)+C(g, h)$ and $C(f, g+h)=C(f, g)+C(f, h)$,

(C3) $\quad C(f a, g)=C(f, g) L_{a}$ and $C(f, g a)=L_{a^{*}} C(f, g)$,

for all $f, g, h \in M$ and $a \in A$. 
Since

$$
\begin{aligned}
L_{B(f, g) a-a^{*} B(f, g)} & =L_{a^{*} B(f, g)+B(f, g) a}-2 L_{a^{*} B(f, g)} \\
& \stackrel{(\mathrm{B} 6)}{=} L_{B(f a, g)+B(f, g a)}-2 L_{a^{*} B(f, g)} \\
& =\left(L_{B(f a, g)}+L_{B(f, g a)}\right)-2 L_{a^{*} B(f, g)} \\
& =\left(L_{B(f a, g)}-L_{a^{*} B(f, g)}\right)+\left(L_{B(f, g a)}-L_{a^{*} B(f, g)}\right) \\
& \stackrel{(\mathrm{B} 2)}{=}\left(L_{B(f a, g)}-L_{a^{*} B(f, g)}\right)+\left(L_{B(g a, f)}-L_{a^{*} B(g, f)}\right) \\
& \stackrel{(\mathrm{BC})}{=}\left(C(f, g) L_{a}-L_{a^{*}} C(f, g)\right)+\left(C(g, f) L_{a}-L_{a^{*}} C(g, f)\right) \\
& =(C(f, g)+C(g, f)) L_{a}-L_{a^{*}}(C(f, g)+C(g, f))
\end{aligned}
$$

and considering the uniqueness of the representation from Lemma 1.3 (more specifically, considering Remark 1.4), we obtain $C(f, g)+C(g, f)=L_{B(f, g)}$. If we put $g=f$ and apply $(\mathrm{B} 1)$, we get $C(f, f)=2 L_{Q(f)}$. For all $f, g, h \in M$ and $a \in A$,

$$
\begin{aligned}
L_{B(f a, g+h)}- & L_{a^{*} B(f, g+h)} \\
& \stackrel{(\mathrm{B} 3)}{=}\left(L_{B(f a, g)}+L_{B(f a, h)}\right)-\left(L_{a^{*} B(f, g)}+L_{a^{*} B(f, h)}\right) \\
& =\left(L_{B(f a, g)}-L_{a^{*} B(f, g)}\right)+\left(L_{B(f a, h)}-L_{a^{*} B(f, h)}\right) \\
& \stackrel{(\mathrm{BC})}{=}\left(C(f, g) L_{a}-L_{a^{*}} C(f, g)\right)+\left(C(f, h) L_{a}-L_{a^{*}} C(f, h)\right) \\
& =(C(f, g)+C(f, h)) L_{a}-L_{a^{*}}(C(f, g)+C(f, h)) .
\end{aligned}
$$

Therefore, the mapping $C$ is additive in the second argument. Analogously we get additivity in the first argument.

Because

$$
\begin{aligned}
& L_{B((f a) b, g)}-L_{b^{*} B(f a, g)} \\
& \quad=\left(L_{B(f(a b), g)}-L_{(a b)^{*} B(f, g)}\right)+\left(L_{(a b)^{*} B(f, g)}-L_{b^{*} B(f a, g)}\right) \\
& =\left(L_{B(f(a b), g)}-L_{(a b)^{*} B(f, g)}\right)-L_{b^{*}}\left(L_{B(f a, g)}-L_{a^{*} B(f, g)}\right) \\
& \quad \stackrel{(\mathrm{BC})}{=}\left(C(f, g) L_{a b}-L_{(a b)^{*}} C(f, g)\right)-L_{b^{*}}\left(C(f, g) L_{a}-L_{a^{*}} C(f, g)\right) \\
& =\left(C(f, g) L_{a}\right) L_{b}-L_{b^{*}}\left(C(f, g) L_{a}\right),
\end{aligned}
$$

we conclude that $C(f a, g)=C(f, g) L_{a}$. Further,

$$
\begin{aligned}
C(f, g a) & \stackrel{(\mathrm{C} 1)}{=} L_{B(g a, f)}-C(g a, f)=L_{B(g a, f)}-C(g, f) L_{a} \\
& \stackrel{(\mathrm{BC})}{=} L_{a^{*} B(g, f)}-L_{a^{*}} C(g, f)=L_{a^{*}}\left(L_{B(g, f)}-C(g, f)\right) \\
& \stackrel{(\mathrm{C} 1)}{=} L_{a^{*}} C(f, g) .
\end{aligned}
$$


Finally, it is sufficient to define the mapping $S: M \times M \rightarrow L_{\mathrm{DC}}(A)$ by

$$
S(f, g)=\frac{1}{2} C(g, f) .
$$

We have

$$
\begin{aligned}
& S\left(f\left(i e_{\alpha}\right), g\right) a=L_{-i e_{\alpha}^{*}} S(f, g) a=-i e_{\alpha}^{*}(S(f, g) a) \rightarrow-i S(f, g) a, \\
& S\left(g, f\left(i e_{\alpha}\right)\right) a=S(g, f) L_{i e_{\alpha}} a=S(g, f)\left(i e_{\alpha} a\right) \rightarrow i S(g, f) a,
\end{aligned}
$$

which is obtained using the fact that the mapping $S(g, f)$ is a bounded linear operator (Remark 1.2). Thus

$$
\begin{aligned}
S(f, g) a= & \frac{1}{2}(S(f, g) a+S(g, f) a)+\frac{i}{2}(-i S(f, g) a+i S(g, f) a) \\
= & \frac{1}{4}(2 S(f, g) a+2 S(g, f) a) \\
& +\frac{i}{4} \lim _{\alpha}\left(2 S\left(f\left(i e_{\alpha}\right), g\right) a+2 S\left(g, f\left(i e_{\alpha}\right)\right) a\right) \\
= & \frac{1}{4}(S(f+g, f+g) a-S(f-g, f-g) a) \\
& +\frac{i}{4} \lim _{\alpha}\left(S\left(f\left(i e_{\alpha}\right)+g, f\left(i e_{\alpha}\right)+g\right) a-S\left(f\left(i e_{\alpha}\right)-g, f\left(i e_{\alpha}\right)-g\right) a\right) \\
= & \frac{1}{4}\left(L_{Q(f+g)} a-L_{Q(f-g)} a\right)+\frac{i}{4} \lim _{\alpha}\left(L_{Q\left(f\left(i e_{\alpha}\right)+g\right)} a-L_{Q\left(f\left(i e_{\alpha}\right)-g\right)} a\right) \\
= & \frac{1}{4}(Q(f+g) a-Q(f-g) a) \\
& +\frac{i}{4} \lim _{\alpha}\left(Q\left(f\left(i e_{\alpha}\right)+g\right) a-Q\left(f\left(i e_{\alpha}\right)-g\right) a\right) .
\end{aligned}
$$

It remains to answer the question under what (necessary and sufficient) conditions a quadratic functional taking values in $A$ can be represented by a sesquilinear form also taking values in $A$. We shall need the following technical lemma:

Lemma 2.6. Let $A, M, Q$ and $S$ be as in Theorem 2.1. If for every pair $(f, g) \in M \times M$ there exists (a unique) $u_{f, g} \in A$ such that

$$
u_{f, g} a=S(f, g) a \quad \text { for every } a \in A,
$$

then the mapping $(f, g) \mapsto u_{f, g}$ is a unique A-valued sesquilinear form on $M \times M$ satisfying $u_{f, f}=Q(f)$ for every $f \in M$.

Proof. Since $S$ is a biadditive form, the mapping $(f, g) \mapsto u_{f, g}$ is also biadditive. Further,

$u_{f a, g} b=S(f a, g) b=L_{a^{*}} S(f, g) b=a^{*}(S(f, g) b)=a^{*} u_{f, g} b$ for every $b \in A$, so

$$
u_{f a, g}=a^{*} u_{f, g}
$$


and similarly

$$
u_{f, g a} b=S(f, g a) b=S(f, g) L_{a} b=S(f, g)(a b)=u_{f, g} a b \text { for every } b \in A,
$$

so

$$
u_{f, g a}=u_{f, g} a .
$$

Finally,

$$
u_{f, f} a=S(f, f) a=L_{Q(f)} a=Q(f) a \quad \text { for every } a \in A,
$$

which yields

$$
u_{f, f}=Q(f) .
$$

Proposition 2.7. Let $A,\left\{e_{\alpha}\right\}, M$ and $Q$ be as in Theorem 2.1. There exists a unique sesquilinear form $S: M \times M \rightarrow A$ representing $Q$, that is, a unique sesquilinear form satisfying $S(f, f)=Q(f)$ for every $f \in M$, if and only if for all $f, g \in M$ the limit $\lim _{\alpha} Q\left(f\left(i e_{\alpha}\right)+g\right) \in A$ exists. In that case, $S(f, g)=\frac{1}{4}(Q(f+g)-Q(f-g))+\frac{i}{4} \lim _{\alpha}\left(Q\left(f\left(i e_{\alpha}\right)+g\right)-Q\left(f\left(i e_{\alpha}\right)-g\right)\right)$. If $Q(f)^{*}=Q(f)$ for every $f \in M$, then $S(f, g)^{*}=S(g, f)$ for all $f, g \in M$.

Proof. Assume that, for all $f, g \in M$, the $\operatorname{limit}_{\lim _{\alpha}}\left(Q\left(f\left(i e_{\alpha}\right)+g\right)\right.$ exists and that it is an element of $A$. The mapping from $M \times M$ to $L_{\mathrm{DC}}(A)$ obtained in Theorem 2.1 will be denoted by $\widetilde{S}$. Define

$$
S(f, g)=\frac{1}{4}(Q(f+g)-Q(f-g))+\frac{i}{4} \lim _{\alpha}\left(Q\left(f\left(i e_{\alpha}\right)+g\right)-Q\left(f\left(i e_{\alpha}\right)-g\right)\right) \text {. }
$$

For all $f, g \in M$ we have $S(f, g) \in A$ and $S(f, g) a=\widetilde{S}(f, g) a$ for every $a \in A$. Lemma 2.6 implies that $S$ is the unique sesquilinear form from $M \times M$ to $A$ with $S(f, f)=Q(f)$ for every $f \in M$. If $B: M \times M \rightarrow A$ is defined as in the proof of Theorem 2.1, then

$$
S(f, g)=\frac{1}{4} B(f, g)+\frac{i}{4} \lim _{\alpha} B\left(f\left(i e_{\alpha}\right), g\right) .
$$

If $Q(f)^{*}=Q(f)$ for every $f \in M$, then $B(f, g)^{*}=B(f, g)$ for all $f, g \in M$. Now we have

$$
\begin{aligned}
S(f, g)^{*}-S(g, f)= & \left(\frac{1}{4} B(f, g)-\frac{i}{4} \lim _{\alpha} B\left(f\left(i e_{\alpha}\right), g\right)\right) \\
& -\left(\frac{1}{4} B(g, f)+\frac{i}{4} \lim _{\alpha} B\left(g\left(i e_{\alpha}\right), f\right)\right) \\
\stackrel{(\mathrm{B} 2)}{=} & -\frac{i}{4} \lim _{\alpha}\left(B\left(f\left(i e_{\alpha}\right), g\right)+B\left(f, g\left(i e_{\alpha}\right)\right)\right) \\
\stackrel{(\mathrm{B} 6)}{=} & -\frac{i}{4} \lim _{\alpha}\left(-i e_{\alpha}^{*} B(f, g)+i B(f, g) e_{\alpha}\right)=0,
\end{aligned}
$$

hence $S(f, g)^{*}=S(g, f)$ for all $f, g \in M$. 
Conversely, if $Q$ can be represented by a sesquilinear form $S: M \times M$ $\rightarrow A$, then

$$
\begin{aligned}
Q\left(f\left(i e_{\alpha}\right)+g\right) & =S\left(f\left(i e_{\alpha}\right)+g, f\left(i e_{\alpha}\right)+g\right) \\
& =e_{\alpha}^{*} S(f, f) e_{\alpha}+i S(g, f) e_{\alpha}-i e_{\alpha}^{*} S(f, g)+S(g, g) \\
& \rightarrow S(f, f)+S(g, g)+i S(g, f)-i S(f, g) \in A .
\end{aligned}
$$

As we are going to see, when $M$ is equipped with the structure of a complex vector space compatible with the structure of $A$, then each $A$ valued quadratic functional on $M$ can be represented by a unique $A$-valued sesquilinear form on $M \times M$. This result can be considered a generalization of the Jordan-von Neumann type theorem for modules over $\mathrm{C}^{*}$-algebras ([18, Theorem 5.1]) to modules over a larger class of algebras. Let us note that the above mentioned theorem is going to be generalized (in the case of $\mathrm{C}^{*}$-algebras as well as in the case of $\mathrm{H}^{*}$-algebras) in Section 4 in yet another sense.

Proposition 2.8. Let $A$ be as in Theorem 2.1. Let the right module $M$ over $A$ be a complex vector space compatible with the structure of $A$. For each quadratic functional $Q: M \rightarrow A$ there exists a unique sesquilinear form $S: M \times M \rightarrow A$ such that $S(f, f)=Q(f)$ for every $f \in M$. Furthermore,

$$
S(f, g)=\frac{1}{4}(Q(f+g)-Q(f-g))+\frac{i}{4}(Q(i f+g)-Q(i f-g)) .
$$

If $Q(f)^{*}=Q(f)$ for every $f \in M$, then $S(f, g)^{*}=S(g, f)$ for all $f, g \in M$.

Proof. Denote the mapping from $M \times M$ to $L_{\mathrm{DC}}(A)$ obtained in Theorem 2.1 by $\widetilde{S}$. Let $\left\{e_{\alpha}\right\}$ be as in Theorem 2.1. For all $f, g \in M$,

$$
\begin{aligned}
Q\left(f\left(i e_{\alpha}\right)\right. & +g) a-Q\left(f\left(i e_{\alpha}\right)-g\right) a=L_{Q\left(f\left(i e_{\alpha}\right)+g\right)} a-L_{Q\left(f\left(i e_{\alpha}\right)-g\right)} a \\
= & \widetilde{S}\left(f\left(i e_{\alpha}\right)+g, f\left(i e_{\alpha}\right)+g\right) a-\widetilde{S}\left(f\left(i e_{\alpha}\right)-g, f\left(i e_{\alpha}\right)-g\right) a \\
= & 2 \widetilde{S}\left(f\left(i e_{\alpha}\right), g\right) a+2 \widetilde{S}\left(g, f\left(i e_{\alpha}\right)\right) a=2 \widetilde{S}\left((i f) e_{\alpha}, g\right) a+2 \widetilde{S}\left(g,(i f) e_{\alpha}\right) a \\
= & 2 L_{e_{\alpha}^{*}} \widetilde{S}(i f, g) a+2 \widetilde{S}(g, i f) L_{e_{\alpha}} a=2 e_{\alpha}^{*} \widetilde{S}(i f, g) a+2 \widetilde{S}(g, i f)\left(e_{\alpha} a\right) \\
\rightarrow & 2 \widetilde{S}(i f, g) a+2 \widetilde{S}(g, i f) a=\widetilde{S}(i f+g, i f+g) a-\widetilde{S}(i f-g, i f-g) a \\
= & L_{Q(i f+g)} a-L_{Q(i f-g)} a=Q(i f+g) a-Q(i f-g) a .
\end{aligned}
$$

Thus,

$$
\widetilde{S}(f, g) a=\frac{1}{4}(Q(f+g) a-Q(f-g) a)+\frac{i}{4}(Q(i f+g) a-Q(i f-g) a) .
$$

That is, if we define $S(f, g)$ as in the statement of the proposition, then $S(f, g) a=\widetilde{S}(f, g) a$ for every $a \in A$. According to Lemma 2.6, $S$ is the unique $A$-valued sesquilinear form representing $Q$. 
Note that if $B: M \times M \rightarrow A$ is as in the proof of Theorem 2.1, then

$$
S(f, g)=\frac{1}{4} B(f, g)+\frac{i}{4} B(i f, g) .
$$

Since

$$
\begin{aligned}
B(i f, g) & =\lim _{\alpha} e_{\alpha}^{*} B(i f, g) e_{\alpha} \stackrel{(\mathrm{B} 4)}{=} \lim _{\alpha} B\left((i f) e_{\alpha}, g e_{\alpha}\right) \\
& =\lim _{\alpha} B\left(f\left(i e_{\alpha}\right),(-i g)\left(i e_{\alpha}\right)\right) \stackrel{(\mathrm{B} 4)}{=} \lim _{\alpha}\left(i e_{\alpha}\right)^{*} B(f,-i g)\left(i e_{\alpha}\right) \\
& =\lim _{\alpha} e_{\alpha}^{*} B(f,-i g) e_{\alpha}=B(f,-i g) \stackrel{(\mathrm{B} 3)}{=}-B(f, i g),
\end{aligned}
$$

the assumption $Q(f)^{*}=Q(f)$, for every $f \in M$, implies

$$
\begin{gathered}
S(f, g)^{*}-S(g, f)=\left(\frac{1}{4} B(f, g)-\frac{i}{4} B(i f, g)\right)-\left(\frac{1}{4} B(g, f)+\frac{i}{4} B(i g, f)\right) \\
\stackrel{(\mathrm{B} 2)}{=}-\frac{i}{4}(B(i f, g)+B(f, i g))=0,
\end{gathered}
$$

so $S(f, g)^{*}=S(g, f)$ for all $f, g \in M$.

The closeness of the representability problem in this case to the one concerning modules over complex *-algebras with an identity is evident: the corresponding formula in the case of modules over unital complex $*$-algebras is

$$
S(f, g)=\frac{1}{4}(Q(f+g)-Q(f-g))+\frac{i}{4}(Q(i f+g)-Q(i f-g))
$$

([13, Corollary 4]). Let us mention that, for simplicity of the proof of the representation theorem in [18] (Theorem 4.3), Zalar introduced a two-parameter generalization of a Jordan $*$-derivation called a Jordan $*$-derivation pair (for his motivation see the beginning of Section 2 in [18]). The explicit formula recovering the sesquilinear form that represents a given quadratic functional indicates why we have decided to deal with a one-parameter mapping, that is, with a Jordan $*$-derivation. Namely, the "real" part of the sesquilinear form obtained is the same as in the unital case. However, the "imaginary" part is a source of problems (because of the elements if) - this is the place where we need a parameter from a Jordan $*$-derivation.

\section{Quadratic functionals on modules over standard operator} algebras. We denote by $\mathcal{H}$ a (real or complex) Hilbert space. A standard operator algebra is a (not necessarily self-adjoint) subalgebra of $\mathbf{B}(\mathcal{H})$ (the algebra of all bounded linear operators on $\mathcal{H}$ ) that contains the ideal $\mathbf{F}(\mathcal{H})$ of finite rank operators. For example, $\mathbf{H S}(\mathcal{H})$ and $\mathbf{K}(\mathcal{H})$ (the algebra of all Hilbert-Schmidt operators on $\mathcal{H}$ and the algebra of all compact linear operators on $\mathcal{H}$, respectively) are standard operator algebras. The following 
example, inspired by Molnár's example in [6], is an example of a standard operator algebra that is not self-adjoint.

ExAmple 3.1. Let $\mathcal{H}$ be an infinite-dimensional Hilbert space. Choose $u \in \mathbf{B}(\mathcal{H})$ such that the kernel of $u^{*}$ is infinite-dimensional and such that $u^{*} u=\mathbf{1}$ (concretely, $u$ is as in the Example of [6]). The algebra $A=$ $\mathbf{F}(\mathcal{H})+u \mathbf{B}(\mathcal{H})$ is a standard operator algebra and $u=0+u \cdot \mathbf{1} \in A$. If we assume that $u^{*} \in A$, then there exist $f \in \mathbf{F}(\mathcal{H})$ and $b \in \mathbf{B}(\mathcal{H})$ having the property $u^{*}=f+u b$, that is, $u=f^{*}+b^{*} u^{*}$. Multiplying this by $u^{*}$ from the left, we get $\mathbf{1}=u^{*} f^{*}+u^{*} b^{*} u^{*}$ and $\mathbf{1}-u^{*} b^{*} u^{*}=u^{*} f^{*} \in \mathbf{F}(\mathcal{H})$ follows. However, $\mathbf{1}-u^{*} b^{*} u^{*}$ is the identity operator on the kernel of $u^{*}$, hence it is not a finite rank operator. Therefore, $u^{*} \notin A$.

LEMMA 3.2. Let $A \subseteq \mathbf{B}(\mathcal{H})$ be a standard operator algebra and let $|\cdot|$ be a norm in $\mathcal{H}$. There exists a generalized sequence $\left\{p_{\alpha}\right\}$, consisting of projections in $A$, such that $\lim _{\alpha}\left|p_{\alpha} a p_{\alpha} \xi-a \xi\right|=0$ for every $a \in A$ and every $\xi \in \mathcal{H}$.

Proof. There exists a generalized sequence $\left\{p_{\alpha}\right\}$ consisting of finite rank projections such that $\left|p_{\alpha} \eta-\eta\right| \rightarrow 0$ for every $\eta \in \mathcal{H}$. Thus $\left\{p_{\alpha}\right\} \subseteq \mathbf{F}(\mathcal{H}) \subseteq A$ and for every $a \in A$ and every $\xi \in \mathcal{H}$ we have

$$
\begin{aligned}
\left|p_{\alpha} a p_{\alpha} \xi-a \xi\right| & \leq\left|p_{\alpha} a p_{\alpha} \xi-p_{\alpha} a \xi\right|+\left|p_{\alpha} a \xi-a \xi\right| \\
& =\left|p_{\alpha} a\left(p_{\alpha} \xi-\xi\right)\right|+\left|p_{\alpha} a \xi-a \xi\right| \\
& \leq\left\|p_{\alpha} a\right\| \cdot\left|p_{\alpha} \xi-\xi\right|+\left|p_{\alpha} a \xi-a \xi\right| \\
& \leq\|a\| \cdot\left|p_{\alpha} \xi-\xi\right|+\left|p_{\alpha}(a \xi)-a \xi\right| \rightarrow 0
\end{aligned}
$$

where $\|\cdot\|$ denotes the operator norm on $\mathbf{B}(\mathcal{H})$.

TheOREM 3.3. Let $A \subseteq \mathbf{B}(\mathcal{H}), \operatorname{dim} \mathcal{H}>1$, be a standard operator algebra and let $M$ be a right module over $A$. Then for each quadratic functional $Q: M \rightarrow A$ there exists a sesquilinear form $S: M \times M \rightarrow \mathbf{B}(\mathcal{H})$ having the property $S(f, f)=Q(f)$ for every $f \in M$.

Proof. In view of Lemma 3.2, the statements (B1)-(B6) from the proof of Theorem 2.1 hold for any standard operator algebra (in (B5) we consider $B(f+f a, g+g a) \xi$ for an arbitrary $\xi \in \mathcal{H}$ and calculate in the same way). Therefore, for fixed $f, g \in M$, the mapping $J: A \rightarrow A$ given by

$$
J(a)=B(f a, g)-a^{*} B(f, g)
$$

is a Jordan $*$-derivation (as in the proof of Theorem 2.1). We further argue as in the proof of Theorem 2.1 again; we present the proof in full for the sake of completeness. The Theorem in [14] implies that for each Jordan $*$-derivation $J: A \rightarrow \mathbf{B}(\mathcal{H})$ there exists a unique $u \in \mathbf{B}(\mathcal{H})$ such that $J(a)=u a-a^{*} u$ for every $a \in A$. Specifically, there is a unique $u_{f, g} \in \mathbf{B}(\mathcal{H})$ having the property

$$
B(f a, g)-a^{*} B(f, g)=u_{f, g} a-a^{*} u_{f, g} \quad \text { for every } a \in A \text {. }
$$


From

$$
\begin{aligned}
\left(u_{f, g}+\right. & \left.u_{g, f}\right) a-a^{*}\left(u_{f, g}+u_{g, f}\right)=\left(u_{f, g} a-a^{*} u_{f, g}\right)+\left(u_{g, f} a-a^{*} u_{g, f}\right) \\
& =\left(B(f a, g)-a^{*} B(f, g)\right)+\left(B(g a, f)-a^{*} B(g, f)\right) \\
& =\left(B(f a, g)-a^{*} B(f, g)\right)+\left(B(f, g a)-a^{*} B(f, g)\right) \\
& =(B(f a, g)+B(f, g a))-2 a^{*} B(f, g) \\
& =\left(a^{*} B(f, g)+B(f, g) a\right)-2 a^{*} B(f, g)=B(f, g) a-a^{*} B(f, g)
\end{aligned}
$$

we get $u_{f, g}+u_{g, f}=B(f, g)$. In particular, $u_{f, f}=\frac{1}{2} B(f, f)=2 Q(f)$. Furthermore,

$$
\begin{aligned}
B(f a, g+h)-a^{*} B & (f, g+h) \\
= & (B(f a, g)+B(f a, h))-a^{*}(B(f, g)+B(f, h)) \\
= & \left(B(f a, g)-a^{*} B(f, g)\right)+\left(B(f a, h)-a^{*} B(f, h)\right) \\
& =\left(u_{f, g} a-a^{*} u_{f, g}\right)+\left(u_{f, h} a-a^{*} u_{f, h}\right) \\
& =\left(u_{f, g}+u_{f, h}\right) a-a^{*}\left(u_{f, g}+u_{f, h}\right),
\end{aligned}
$$

so $u_{f, g+h}=u_{f, g}+u_{f, h}$. The same method gives us $u_{f+g, h}=u_{f, h}+u_{g, h}$. Since

$$
\begin{aligned}
B & ((f a) b, g)-b^{*} B(f a, g) \\
& =\left(B(f(a b), g)-(a b)^{*} B(f, g)\right)+\left((a b)^{*} B(f, g)-b^{*} B(f a, g)\right) \\
& =\left(B(f(a b), g)-(a b)^{*} B(f, g)\right)-b^{*}\left(B(f a, g)-a^{*} B(f, g)\right) \\
& =\left(u_{f, g}(a b)-(a b)^{*} u_{f, g}\right)-b^{*}\left(u_{f, g} a-a^{*} u_{f, g}\right)=\left(u_{f, g} a\right) b-b^{*}\left(u_{f, g} a\right),
\end{aligned}
$$

we have $u_{f a, g}=u_{f, g} a$. We further obtain

$$
\begin{aligned}
u_{f, g a} & =B(g a, f)-u_{g a, f}=B(g a, f)-u_{g, f} a \\
& =a^{*} B(g, f)-a^{*} u_{g, f}=a^{*}\left(B(g, f)-u_{g, f}\right)=a^{*} u_{f, g} .
\end{aligned}
$$

Finally, we define the mapping $S: M \times M \rightarrow \mathbf{B}(\mathcal{H})$ by

$$
S(f, g)=\frac{1}{2} u_{g, f}
$$

REMARK 3.4. If $A \subseteq \mathbf{B}(\mathcal{H}), \operatorname{dim} \mathcal{H}>1$, is a standard operator algebra, then for every $\xi \in \mathcal{H}$ there exist $a, b \in A$ satisfying $a=a^{*}, a \xi=\xi, b a=0$ and $b b^{*}=a^{2}$. (Namely, it is sufficient to define $a$ as the orthogonal projection on the subspace of $\mathcal{H}$ generated by $\xi$, then to take any $\eta$ orthogonal to $\xi$ having the same norm as $\xi$ and finally to define $b: \mathcal{H} \rightarrow \mathcal{H}$ by $b(\lambda \eta+\varrho)=\lambda \xi$ for every scalar $\lambda$ and every $\varrho$ orthogonal to $\eta$.)

This is in fact the statement of Lemma 14 in [8], carried over from the context of $\mathrm{C}^{*}$-algebras to the context of standard operator algebras. It enables us, arguing as in the proof of Lemma 15 in [8], to conclude that each 
positive definite mapping $Q: M \rightarrow A$, where $M$ is a right module over $A$, satisfying $Q(f a)=a^{*} Q(f) a(f \in M, a \in A)$ also satisfies the parallelogram law. (The possibility of carrying over this result was independently observed by D. Bakić in the case $A=\mathbf{H S}(\mathcal{H}), \operatorname{dim} \mathcal{H}>1$.)

Note that, although it is required for $Q$ to be positive definite, only the fact $Q(f)^{*}=Q(f)$ for every $f \in M$ is used in the proof. Hence, the statement also holds for hermitian mappings. When $\mathcal{H}$ is complex, clearly the assumption that $Q$ is hermitian can be omitted as well.

Finally, a generalization of this result to an arbitrary $\mathrm{H}^{*}$-algebra can be obtained without difficulties (every $\mathrm{H}^{*}$-algebra is the closure of the orthogonal sum of its minimal closed two-sided ideals, each of which is a topologically simple $\mathrm{H}^{*}$-algebra and the $\mathrm{H}^{*}$-algebras $\mathbf{H S}(\mathcal{H})$ are the only topologically simple $\mathrm{H}^{*}$-algebras; see [1]). More precisely, if $A$ is an $\mathrm{H}^{*}$-algebra with no nonzero commutative closed two-sided ideals, $M$ is a right module over $A$ and $Q: M \rightarrow A$ is the mapping that satisfies $Q(f a)=a^{*} Q(f) a$ $(f \in M, a \in A)$, then $Q$ is a quadratic functional. The same is true in the case when $A$ is a $\mathrm{C}^{*}$-algebra having the same property (see the above-mentioned Lemma 15 of [8]).

4. Normed modules over $\mathrm{H}^{*}$-algebras and $\mathrm{C}^{*}$-algebras. The known concept of a Hilbert module over an $\mathrm{H}^{*}$-algebra or a $\mathrm{C}^{*}$-algebra, which arises as a generalization of a complex Hilbert space, can be extended to the concept of a pre-Hilbert module over an $\mathrm{H}^{*}$-algebra or a $\mathrm{C}^{*}$-algebra, that is, to a generalization of a complex pre-Hilbert space.

Let $A$ be an $\mathrm{H}^{*}$-algebra or a $\mathrm{C}^{*}$-algebra. A pre-Hilbert $A$-module is a right module $H$ over $A$ which is equipped with a generalized inner product, that is, with a mapping $[\cdot, \cdot]$ on $H \times H$, which is $\tau(A)$-valued if $A$ is an $\mathrm{H}^{*}$-algebra, or $A$-valued if $A$ is a $\mathrm{C}^{*}$-algebra, having the following properties:

$$
\begin{aligned}
& {[f, g+h]=[f, g]+[f, h] \text { for all } f, g \in H} \\
& {[f, g a]=[f, g] a \text { for all } f, g \in H \text { and } a \in A}
\end{aligned}
$$

$[f, g]^{*}=[g, f]$ for all $f, g \in H$,

$[f, f] \geq 0$ for every $f \in H$, and $[f, f]=0$ implies $f=0$.

If $A$ is an $\mathrm{H}^{*}$-algebra, then for each positive $a \in \tau(A)$ there exists a unique positive element $b \in A$ such that $b^{2}=a$; if $A$ is a $\mathrm{C}^{*}$-algebra, such a $b \in A$ exists for each positive $a \in A$. In both cases, $b$ is called the square root of $a$. Specifically, for every $f \in H$ the square root of $[f, f]$ exists and it is denoted by $|f|$.

Each pre-Hilbert $A$-module $(H,[\cdot, \cdot])$ is a metric space with respect to the metric $d: H \times H \rightarrow \mathbb{C}$ given by $d(f, g)=\||f-g|\|$ (where $\|\cdot\|$ denotes the 
norm in $A)$. If this metric space is complete, then $(H,[\cdot, \cdot])$ is called a Hilbert $A$-module. Completeness and the existence of an approximate identity in $A$ enable us to equip each Hilbert $A$-module with the structure of a complex vector space compatible with the structure of $A$ (see Theorem 1 in [9] or Exercise 15.A in [16], which are basically the same). However, there are pre-Hilbert $A$-modules that lack this property. The following example is a variation of Example 2.5.

EXAMPLE 4.1. Let $A$ be an $\mathrm{H}^{*}$-algebra (or a $\mathrm{C}^{*}$-algebra) such that there exists $x \in A$ with $x v \neq x$ for every $v \in A$. The set $A \times \mathbb{R}$, endowed with coordinatewise addition, is a right module over $A$ under the action

$$
(a, \lambda) b=(a b+\lambda b, 0)
$$

for all $a, b \in A$ and $\lambda \in \mathbb{R}$. Define

$$
M=\{(a, \lambda) \in A \times \mathbb{R}: x a+\lambda x=0\} .
$$

Obviously, $M$ is closed with respect to addition. For all $a, b \in A$ and $\lambda \in \mathbb{R}$, the equality $x a+\lambda x=0$ implies

$$
x(a b+\lambda b)+0 \cdot x=(x a+\lambda x) b=0 \cdot b=0,
$$

so $(a b+\lambda b, 0) \in M$, that is, $(a, \lambda) b \in M$ for every $(a, \lambda) \in M$ and every $b \in A$. Hence, $M$ is a submodule of $A \times \mathbb{R}$. Put $H=(A \times \mathbb{R}) / M$ and define

$$
((a, \lambda)+M)+((b, \mu)+M)=(a+b, \lambda+\mu)+M
$$

for all $a, b \in A$ and $\lambda, \mu \in \mathbb{R}$ and

$$
((a, \lambda)+M) b=(a b+\lambda b, 0)+M
$$

for all $a, b \in A$ and $\lambda \in \mathbb{R}$. Under these operations, $H$ is a right module over $A$. We can equip it with the structure of a pre-Hilbert module over $A$ if we define

$$
[(a, \lambda)+M,(b, \mu)+M]=(x a+\lambda x)^{*}(x b+\mu x)
$$

for all $a, b \in A$ and $\lambda, \mu \in \mathbb{R}$. Assume that $H$ has the structure of a complex vector space compatible with the structure of $A$. Let $\left\{e_{\alpha}\right\}$ be an approximate identity for $A$. We have

$$
(i \cdot((0,1)+M)) e_{\alpha}=((0,1)+M)\left(i e_{\alpha}\right),
$$

that is,

$$
(i \cdot((0,1)+M)) e_{\alpha}=\left(i e_{\alpha}, 0\right)+M .
$$

If $i \cdot((0,1)+M)=(u, \eta)+M \in H$, then

$$
(i \cdot((0,1)+M)) e_{\alpha}=((u, \eta)+M) e_{\alpha}=\left(u e_{\alpha}+\eta e_{\alpha}, 0\right)+M .
$$

Therefore,

$$
\left(u e_{\alpha}+\eta e_{\alpha}-i e_{\alpha}, 0\right) \in M \quad \text { for every } \alpha,
$$


SO

$$
x\left(u e_{\alpha}+\eta e_{\alpha}-i e_{\alpha}\right)=0 \quad \text { for every } \alpha .
$$

After taking limits, we get

$$
x u=(i-\eta) x .
$$

Since $\eta \in \mathbb{R}$, we have $\eta \neq i$. If we define $v=\frac{1}{i-\eta} u \in A$, then $x v=x$, contradicting our assumption. Hence, $H$ is a pre-Hilbert module over an $\mathrm{H}^{*}$-algebra or a $\mathrm{C}^{*}$-algebra $A$ which cannot be equipped with the structure of a complex vector space compatible with the structure of $A$.

Let $(A,\|\cdot\|)$ be an $\mathrm{H}^{*}$-algebra or a $\mathrm{C}^{*}$-algebra. A normed $A$-module is a right module $H$ over $A$ together with a mapping $N: H \rightarrow A$ having the following properties:

(N1) $\quad N(f) \geq 0$ for every $f \in H$,

(N2) $\quad N(f)=0$ implies $f=0$,

(N3) $\quad N(f a)=|N(f) a|$ for every $f \in H$ and every $a \in A$,

(N4) $\quad\|N(f+g)\| \leq\|N(f)\|+\|N(g)\|$ for all $f, g \in H$.

For every $a \in A,|a|$ denotes the square root of $a^{*} a$. It can be easily verified that $\||a|\|=\|a\|$ for every $a \in A$. This equality and axiom (N3) imply $\|N(f a)\|=\|N(f) a\|$ for every $f \in H$ and every $a \in A$, which will be used later.

As before, $H$ is understood to be a module over the ring $A$. Since

$$
N(f a)^{2}=|N(f) a|^{2}=(N(f) a)^{*}(N(f) a)=a^{*} N(f)^{2} a \quad(f \in H, a \in A),
$$

the mapping $Q: H \rightarrow A$ given by $Q(f)=N(f)^{2}$ satisfies $Q(f a)=a^{*} Q(f) a$ for every $f \in H$ and every $a \in A$. If $A$ does not have nonzero commutative closed two-sided ideals, then $Q$ is a quadratic functional according to Remark 3.4 , so the normed $A$-module $(H, N)$ satisfies the parallelogram law

$$
N(f+g)^{2}+N(f-g)^{2}=2 N(f)^{2}+2 N(g)^{2} \quad(f, g \in H) .
$$

The notion of a normed module over an $\mathrm{H}^{*}$-algebra was introduced by B. Zalar in [17]. In that paper a generalization of the classical Jordan-von Neumann theorem was obtained in the form of a characterization of preHilbert modules over $\mathrm{H}^{*}$-algebras among normed modules over $\mathrm{H}^{*}$-algebras via the parallelogram law. However, as we have noticed, in a large class of normed modules over $\mathrm{H}^{*}$-algebras, the parallelogram law holds.

The analogous notion of a Finsler module in the case of $\mathrm{C}^{*}$-algebras was defined by N. C. Phillips and N. Weaver in [8], but requiring that $H$ is a Banach space with respect to the norm $f \mapsto\|N(f)\|$. They proved that every Finsler $A$-module, when $A$ is a $\mathrm{C}^{*}$-algebra without nonzero commutative closed two-sided ideals, arises from a unique Hilbert $A$-module. 
REMARK 4.2. Zalar's definition of a normed module over an $\mathrm{H}^{*}$-algebra also included the following continuity property:

(N5) If $\left\{f_{\alpha}\right\} \subseteq H$ is a generalized sequence such that for all $\varepsilon>0$ there exists $\alpha_{0}$ such that for all $\alpha, \beta \geq \alpha_{0}$ we have $\left\|N\left(f_{\alpha}-f_{\beta}\right)\right\|<\varepsilon$, then $\left\{N\left(f_{\alpha}\right)\right\}$ is a generalized Cauchy sequence in $A$.

In [3] it is proved that this axiom can be omitted. An important role in the proof is played by the inequality (see the proof of [3, Step 7])

$$
\tau\left(N(f)^{2}-N(g)^{2}\right) \leq\|N(f-g)\|(\|N(f)\|+\|N(g)\|) \quad \text { for all } f, g \in H
$$

that holds in any normed module $(H, N)$ over an $\mathrm{H}^{*}$-algebra $(A,\|\cdot\|)$ satisfying the parallelogram law (in light of Remark 3.4, in any normed module over an $\mathrm{H}^{*}$-algebra without nonzero commutative closed two-sided ideals). Using Akemann's theorem from [8], an analogous inequality can be obtained in any normed module $(H, N)$ over an arbitrary $\mathrm{C}^{*}$-algebra $(A,\|\cdot\|)$. Namely, as in [8, Corollary 5], for all $f, g \in H$ and $a \in A$ with $a \geq 0,\|a\| \leq 1$,

$$
\begin{aligned}
\left|\left\|a N(f)^{2} a\right\|-\left\|a N(g)^{2} a\right\|\right| & =\left|\left\|(N(f) a)^{*}(N(f) a)\right\|-\left\|(N(g) a)^{*}(N(g) a)\right\|\right| \\
& =\left|\|N(f) a\|^{2}-\|N(g) a\|^{2}\right| \\
& =|\|N(f) a\|-\|N(g) a\|| \cdot(\|N(f) a\|+\|N(g) a\|) \\
& =|\|N(f a)\|-\|N(g a)\|| \cdot(\|N(f) a\|+\|N(g) a\|) \\
& (\mathrm{N} 4) \\
& \leq\|N(f a-g a)\| \cdot(\|N(f) a\|+\|N(g) a\|) \\
& =\|N(f-g) a\| \cdot(\|N(f) a\|+\|N(g) a\|) \\
& \leq\|N(f-g)\| \cdot(\|N(f)\|+\|N(g)\|) .
\end{aligned}
$$

Finally, Theorem 4 of $[8]$ implies

$$
\left\|N(f)^{2}-N(g)^{2}\right\| \leq\|N(f-g)\| \cdot(\|N(f)\|+\|N(g)\|) \quad \text { for all } f, g \in H .
$$

This inequality and the corresponding one in the case of $\mathrm{H}^{*}$-algebras will be used in the proof of the following lemma.

Lemma 4.3. Let $(A,\|\cdot\|)$ be an $\mathrm{H}^{*}$-algebra or a $\mathrm{C}^{*}$-algebra, without nonzero commutative closed two-sided ideals. Let $(H, N)$ be a normed A-module. If $\left\{e_{\alpha}\right\}$ is an approximate identity for $A$, then, for all $f, g \in H$, a generalized sequence $\left\{N\left(f\left(i e_{\alpha}\right)+g\right)^{2}\right\}$ converges in $(\tau(A), \tau(\cdot))$ if $A$ is an $\mathrm{H}^{*}$-algebra or in $(A,\|\cdot\|)$ if $A$ is a $\mathrm{C}^{*}$-algebra.

Proof. Note that the right sides of the inequalities in Remark 4.2 are equal in the case of $\mathrm{H}^{*}$-algebras and in the case of $\mathrm{C}^{*}$-algebras. Namely, in both cases the right-hand side is equal to

$$
\|N(f-g)\| \cdot(\|N(f)\|+\|N(g)\|) .
$$

Inserting $f\left(i e_{\alpha}\right)+g$ and $f\left(i e_{\beta}\right)+g$ instead of $f$ and $g$ in the above expression, we conclude that for all $\varepsilon>0$ there exists $\alpha_{0}$ such that for all $\alpha, \beta \geq \alpha_{0}$ we 
have

$$
\begin{aligned}
& \left\|N\left(f\left(i e_{\alpha}\right)-f\left(i e_{\beta}\right)\right)\right\| \cdot\left(\left\|N\left(f\left(i e_{\alpha}\right)+g\right)\right\|+\left\|N\left(f\left(i e_{\beta}\right)+g\right)\right\|\right) \\
& \quad=\left\|N\left(f\left(i e_{\alpha}-i e_{\beta}\right)\right)\right\| \cdot\left(\left\|N\left(f\left(i e_{\alpha}\right)+g\right)\right\|+\left\|N\left(f\left(i e_{\beta}\right)+g\right)\right\|\right) \\
& \quad \stackrel{(\mathrm{N} 4)}{\leq}\left\|N\left(f\left(i e_{\alpha}-i e_{\beta}\right)\right)\right\| \cdot\left(\left\|N\left(f\left(i e_{\alpha}\right)\right)\right\|+\left\|N\left(f\left(i e_{\beta}\right)\right)\right\|+2\|N(g)\|\right) \\
& \quad=\left\|N(f)\left(i e_{\alpha}-i e_{\beta}\right)\right\| \cdot\left(\left\|N(f)\left(i e_{\alpha}\right)\right\|+\left\|N(f)\left(i e_{\beta}\right)\right\|+2\|N(g)\|\right) \\
& \quad=\left\|N(f) e_{\alpha}-N(f) e_{\beta}\right\| \cdot\left(\left\|N(f) e_{\alpha}\right\|+\left\|N(f) e_{\beta}\right\|+2\|N(g)\|\right)<\varepsilon .
\end{aligned}
$$

Thus the left sides of the inequalities from Remark 4.2 are also less than $\varepsilon$. If $A$ is an $\mathrm{H}^{*}$-algebra, this implies that $\left\{N\left(f\left(i e_{\alpha}\right)+g\right)^{2}\right\}$ is a generalized Cauchy sequence in $(\tau(A), \tau(\cdot))$ and thus converges. If $A$ is a $\mathrm{C}^{*}$-algebra, then $\left\{N\left(f\left(i e_{\alpha}\right)+g\right)^{2}\right\}$ is a generalized Cauchy sequence in $(A,\|\cdot\|)$ and is therefore convergent.

THEOREM 4.4. Let $A$ be an $\mathrm{H}^{*}$-algebra or a $\mathrm{C}^{*}$-algebra, without nonzero commutative closed two-sided ideals. Then the class of normed A-modules coincides with the class of pre-Hilbert A-modules.

Proof. Let $(H,[\cdot, \cdot])$ be a pre-Hilbert $A$-module. If we put $N(f)=|f|$, then $(H, N)$ is a normed $A$-module. When $A$ is an $\mathrm{H}^{*}$-algebra, this statement is in fact Theorem 12 in [17]. When $A$ is a $\mathrm{C}^{*}$-algebra, axioms (N1)-(N3) can be verified in the same way as in the case of an $\mathrm{H}^{*}$-algebra. Axiom (N4) can be obtained by simple calculation using the Cauchy-Schwarz inequality that holds in any pre-Hilbert module over a $\mathrm{C}^{*}$-algebra (e.g. [16, Corollary 15.1.4]).

Conversely, let $(H, N)$ be a normed $A$-module and let $\left\{e_{\alpha}\right\}$ be an approximate identity for $A$. The quadratic functional $Q$ defined by $Q(f)=N(f)^{2}$ for every $f \in H$ is $\tau(A)$-valued if $A$ is an $\mathrm{H}^{*}$-algebra, or $A$-valued if $A$ is a $\mathrm{C}^{*}$-algebra. Lemma 4.3 implies that $Q$ satisfies the assumption of Proposition 2.7. Therefore there exists a sesquilinear form $S$ on $H \times H$, with values in $\tau(A)$ if $A$ is an $\mathrm{H}^{*}$-algebra or in $A$ if $A$ is a $\mathrm{C}^{*}$-algebra, satisfying $S(f, f)=Q(f)$ for every $f \in H$. Note that $S(f, g)^{*}=S(g, f)$ for all $f, g \in H$ since $Q(f)^{*}=Q(f)$ for every $f \in H$. If we define $[f, g]=S(f, g)$ for all $f, g \in H$, then axioms (H1)-(H3) obviously hold. Furthermore,

$$
[f, f]=S(f, f)=Q(f)=N(f)^{2}=N(f)^{*} N(f) \geq 0
$$

and

$$
[f, f]=0 \Rightarrow N(f)^{*} N(f)=0 \Rightarrow N(f)=0 \Rightarrow f=0 .
$$

Hence $(H,[\cdot, \cdot])$ is a pre-Hilbert $A$-module. 
This result is, in fact, obtained by combining the algebraic approach from [17] with the analytic approach from [8] and it generalizes Zalar's main theorem in [17] in the case of $\mathrm{H}^{*}$-algebras and Corollary 18 in [8] in the case of $\mathrm{C}^{*}$-algebras (compare with Theorem 5.1 in [18]). Let us mention that for the existence of a unique generalized inner product representing a Finsler norm, when the parallelogram law holds, Proposition 2.8 can be used.

Acknowledgments. The author would like to thank Professor Vladimir M. Manuilov for drawing the author's attention to the paper [8], Professor Damir Bakić for his useful suggestions, and the referee whose comments helped improve the original manuscript.

\section{References}

[1] W. Ambrose, Structure theorems for a special class of Banach algebras, Trans. Amer. Math. Soc. 57 (1945), 364-386.

[2] M. Brešar and B. Zalar, On the structure of Jordan *-derivations, Colloq. Math. 63 (1992), 163-171.

[3] D. Ilišević, On redundance of one of the axioms of a generalized normed space, Glasnik Mat. 37 (57) (2002), 133-139.

[4] -, A new approach to the Jordan-von Neumann type theorem for pre-Hilbert modules over $H^{*}$-algebras, in: Functional Analysis VII (Dubrovnik, 2001), Univ. of Århus, Dept. Math. Sci., Århus, 2002, 121-128.

[5] C. N. Kellogg, Centralizers and $H^{*}$-algebras, Pacific J. Math. 17 (1966), 121-129.

[6] L. Molnár, Jordan *-derivation pairs on a complex *-algebra, Aequationes Math. 54 (1997), 44-55.

[7] G. J. Murphy, $C^{*}$-algebras and Operator Theory, Academic Press, Boston, 1990.

[8] N. C. Phillips and N. Weaver, Modules with norms which take values in a $C^{*}$-algebra, Pacific J. Math. 185 (1998), 163-181.

[9] P. P. Saworotnow, A generalized Hilbert space, Duke Math. J. 35 (1968), 191-197.

[10] -, Trace-class and centralizers of an $H^{*}$-algebra, Proc. Amer. Math. Soc. 26 (1970), 101-104.

[11] P. P. Saworotnow and J. C. Friedell, Trace-class for an arbitrary $H^{*}$-algebra, ibid., 95-100.

[12] P. Šemrl, Quadratic functionals and Jordan *-derivations, Studia Math. 97 (1991), $157-165$.

[13] —, Quadratic and quasi-quadratic functionals, Proc. Amer. Math. Soc. 119 (1993), 1105-1113.

[14] —, Jordan *-derivations of standard operator algebras, ibid. 120 (1994), 515-518.

[15] J. F. Smith, The p-classes of an $H^{*}$-algebra, Pacific J. Math. 42 (1972), 777793.

[16] N. E. Wegge-Olsen, K-theory and $C^{*}$-algebras. A Friendly Approach, Oxford Univ. Press, New York, 1993.

[17] B. Zalar, Jordan-von Neumann theorem for Saworotnow's generalized Hilbert space, Acta Math. Hungar. 69 (1995), 301-325. 
[18] B. Zalar, Jordan *-derivation pairs and quadratic functionals on modules over *rings, Aequationes Math. 54 (1997), 31-43.

Department of Mathematics

University of Zagreb

Bijenička 30

P.O. Box 335

10002 Zagreb, Croatia

E-mail: ilisevic@math.hr

Received March 21, 2003

Revised version May 30, 2005 